\title{
Unintentional Accidents among Children: Associated Factors with moderate /severe forms
}

\author{
Assia Mouad ${ }^{1-2}$, Fadoua Boughaleb ${ }^{1-2}$, Loubna Aqqaoui ${ }^{1-2-3-4}$, Toualouth Lafia ${ }^{1-2,5}$, Aya Skiredj ${ }^{1-2}$, Mounir Erraji ${ }^{1-2}$, Fouad \\ Ettayebi $^{1-2}$, Houda Oubejja $\mathrm{a}^{1-2-3-4}$ \\ 1- Department of pediatric surgical emergencies, children's hospital of Rabat, Morocco. \\ 2- Faculty of medecine and pharmacy, University Mohammed V, Rabat, Morocco. \\ 3- Laboratory of Genetic and Biometry, faculty of science, university Ibn Tofail Kenitra, Morocco. \\ 4- Laboratory of epidemiology, clinical research and Biostatistics, faculty of medecine and pharmacy, University Mohammed V, \\ Rabat. \\ 5- Ministry of health, Republic of Benin
}

\section{Abstract}

Unintentional accidents are very common in children and are the main reason for consultation and hospitalization in paediatric surgery. Aim: To determine the factors associated with unintentional accidents resulting in hospitalization. Material and methods: This is a cross sectional study carried out in the Paediatric Surgical Emergency Department of the Children's Hospital of Rabat over a period of 4 years. We processed 1205 children's files; the data was collected and analysed by Jamovi. Results: for the 545 files selected, the median was 8 years $[4 ; 12]$ and boys were predominant $(70.4 \%)$. Sociodemographic factors were represented by low health insurance. In $95.1 \%$ of cases, the family lived in a house $(50.1 \%)$ or an apartment $(45 \%)$ compared to $4.9 \%$ who had insanitary house. For parents' level of education, fathers had a high school education (43.6\%) or never attended school $(16.9 \%)$, compared to $32.9 \%$ of mothers who did not attend school. No adult was present during the accident $(54.6 \%)$. The accident occurred on weekdays $(73.2 \%)$, especially on winter and summer $(41.6 \%$ and $33.8 \%)$. The main lesions were fractures $(53 \%)$. The lesions involved the limbs $(52.5 \%)$ and the head $(30.2 \%)$ and were multiple in $6.5 \%$ of cases. The outcome was simple in $96.1 \%$.

Keywords: Child. Unintentional Accident. Associated factors. Morocco.

\section{Introduction:}

Unintentional injuries consist on the subset of injuries for which there is no evidence of predetermined intent. They include everyday life injuries (EDL) as well as road traffic accidents (RTA), and work accident. Injuries are defined as damage to a person caused by an acute transfer of mechanical, thermal, electrical, chemical, or radiation energy or by the sudden absence of heat or oxygen [1]. Throughout the world unintentional injury in children is a very important public health issue [2].

Unintentional accidents are one of the most frequent reasons for consultation among children. They can affect all children backgrounds and all age groups. [3].

Unintentional injuries in childhood are serious health problems in modern society. They not only result in great economic loss and take many medical resources, but also cause immeasurable psychological hurt to children themselves and their families. [4].

The aim of this study is to underline the epidemiological profile of children with unintentional injuries, resulting in hospitalization, and to describe the factors associated with a moderate/ severe form.

A better understanding of the number, circumstances of unintentional accidents in children, as well as the nature and severity of the injuries is a fundamental step in helping to understand these accidents, the mortality and morbidity of which are very important,

\section{Material and methods}

The Children's Hospital of Rabat is a tertiary care hospital that is one of the ten health facilities of the Ibn Sina Hospital Center. It is organized in several sectors: emergency department, intensive care unit, hospitalization services and technical platform (Radiology, laboratory of biology and anatomopathology).

A cross-sectional, descriptive study was conducted in 4 years (2016-2019) in the pediatric surgical emergencies (PSE) department, children's hospital of Rabat, Morocco. We included patients less than 15 years, who presented unintentional accidents. Trauma related to poisoning and drowning, as well as those related to an intentional injury were excluded. Verbal and informed consent was obtained from at least one of the parents.

The data were collected using a questionnaire fulfilled by the resident of pediatric surgery, and 
completed if necessary from the patient's file. The demographic, socioeconomic and injury characteristics were listed. The patients were divided into groups: Victims of traffic accidents, and those who have everyday life accidents and also into age groups. We also categorized the patients according to their lesion, thus the head trauma was defined as damage to the scalp, skull, or brain. A Polytrauma is a patient with one or more traumatic injuries, at least one of which is life threatening. Moreover, the lesions were classified into two groups: minor severity and moderate to high severity.

Data were analyzed using JAMOVI Software 1.6.23. [5]. Chi square test was used to compare variable as well as a binomial logistic regression.

\section{Results}

During 4 years, 1245 patients consulted for unintentional accidents of which 545 files were selected. Almost $64 \%$ of patients are above 6 years old (Figure 1), and the male predominance is around $70.4 \%$. The median age was 8 years $[4 ; 12]$. The sociodemographic variables were represented by low health insurance (AMO: $30.4 \%$ and RAMED: $46.5 \%$ ). In $95.1 \%$ of cases, the family lived in a house $(50.1 \%)$ compared to $4.9 \%$ who had unsanitary housing. The location of accident is represented in figure 2 . Around $80 \%$ of accident is related to everyday life injuries, especially falls. The figure 3 underlines the fall's circumstances, which occurred mostly from ground level. For parents' level of education, fathers had a high school education $(43.6 \%)$ or never attended school (16.9\%), compared to $32.9 \%$ of mothers who did not attend school (Figure 4). No adult was present during the accident in $54.6 \%$ of the children. Regarding the time of occurrence of the accident, it was mostly on weekdays $(73.2 \%)$, especially on winter and summer (41.6\% and 33.8\%). Most accidents happened between $8 \mathrm{am} 4 \mathrm{pm}$ (51\%) versus $32 \%$ between $5 \mathrm{pm}$ and $8 \mathrm{pm}$ (Figure 5).

In terms of injuries, the lesions involved the limbs $(52.5 \%)$ followed by the head $(30.2 \%)$ and were multiple in $15 \%$ of cases (Table 2). Moreover, the fractures were encountered in $53 \%$ of cases. Most patients had no complication (96.1\%).

On the other hand, in a univariate logistic regression model, there were some factors associated to moderate /severe forms of the lesions. Indeed, the age $(\mathrm{OR}=0,949$, IC à $95 \%$ : 0,903 $0,997, p=0.036)$, the absence of an adult during the accident $(\mathrm{OR}=0.566$, IC à 95\%: 0.352-0.911, $\mathrm{p}=0.019$ ), and the part of body injured, especially head trauma, multiple lesions and trunk lesions are statistically significant. Besides, the gender, the family size, the number of siblings and the type of trauma are not associated to severity. In multivariate analysis, adjusting on the factors above mentioned, only the parts of body injured were associated to a moderate and severe form (Table 3 )

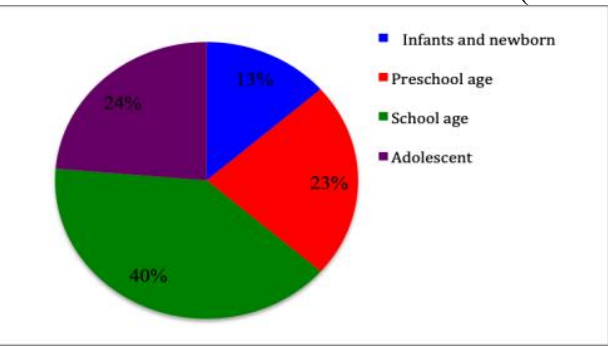

Figure 1: Pie graph showing age group children victims of unintentional accident, PSE department, children's hospital of Rabat.

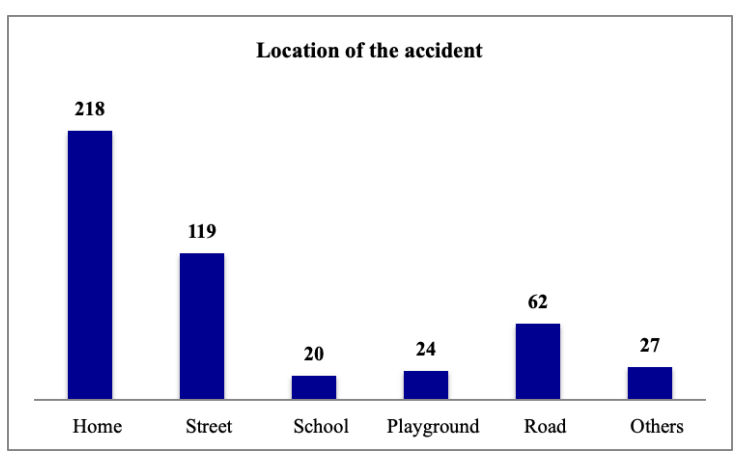

Figure 2: Bar graph showing the location of the unintentional accident, PSE department, children's hospital of Rabat.

Table 1: General characteristics of children victims of unintentional accidents, PSE department, children's hospital of Rabat

\begin{tabular}{lcc}
\hline Characteristics & $n(545)$ & $\%$ \\
\hline Schooling & 139 & 25.9 \\
No & 398 & 74.1 \\
Yes & & \\
School level & 138 & 25.8 \\
Unschooled & 39 & 7.3 \\
Kindergarten & 212 & 39.7 \\
Primary & 117 & 21.9 \\
Middle school & 28 & 5.2 \\
High school & & \\
Area & 431 & 85.5 \\
Urban & 73 & 14.5 \\
Rural & & \\
Dwelling & 247 & 50.1 \\
House & 222 & 45.0 \\
Apartment & 4.9 \\
Shantytown & & \\
\hline
\end{tabular}


Table 2: Injuries characteristics of children victims of unintentional accidents, PSE department, children's hospital of Rabat

\begin{tabular}{lcc}
\hline Characteristics & $n$ & $\%$ \\
& $(545)$ & \\
\hline General Circumstances & 364 & 68.0 \\
Fall & 22 & 4.1 \\
Burns & 105 & 19.6 \\
Collision & 44 & 8.2 \\
Other & & \\
Injured body part (IBP) & 157 & 30.2 \\
Head & 10 & 1.9 \\
Thoraco-abdominal & 80 & 15 \\
Multiple injuries & 273 & 52.5 \\
Limbs & & \\
\hline
\end{tabular}

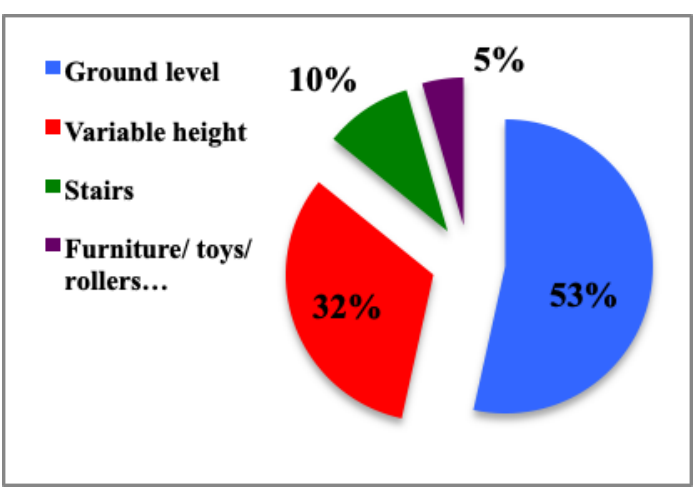

Figure 3: Pie graph showing the fall's circumstances in children victims of unintentional accident, PSE department, children's hospital of Rabat.

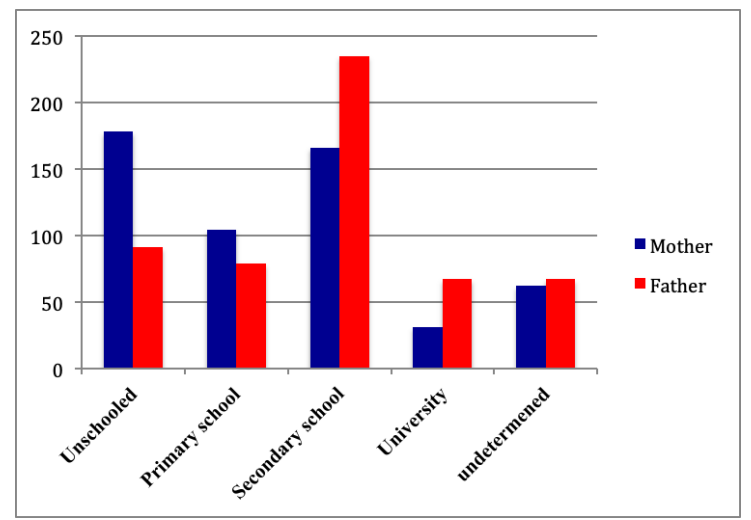

Figure 4: Bar graph showing the parent's educational level, PSE department, children's hospital of Rabat.

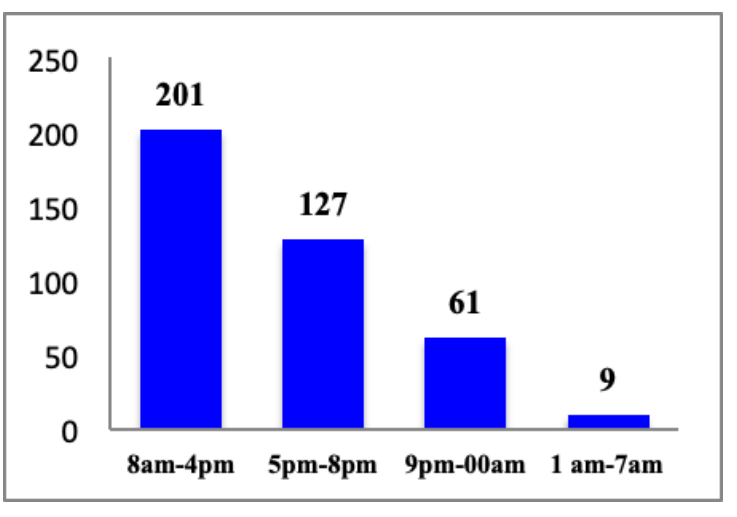

Figure 5: Bar graph showing the time of the unintentional accident by interval, unintentional accident, PSE department, children's hospital of Rabat. 
Table 3: Factors associated to moderate /severe forms of unintentional injuries among children, PSE department, children's hospital of Rabat.

\begin{tabular}{lcccccc}
\hline \multicolumn{1}{c}{ Independant variable } & \multicolumn{3}{c}{ Univariate analysis } & \multicolumn{3}{c}{ Multivariate analyis } \\
\cline { 2 - 7 } Age & OR & IC 95\% & P & OR & IC 95\% & P \\
Gender : Boy /Girl & 0.949 & $0.903-0.997$ & 0.036 & 0.955 & $0.8612-1.059$ & 0.386 \\
Adult present: No/yes & 0.707 & $0.448-1.115$ & 0.136 & 1.014 & $0.4901-2.099$ & 0.970 \\
Schooled : No/yes & 0.566 & $0.352-0.911$ & 0.019 & 0.761 & $0.3790-1.529$ & 0.444 \\
Parts of body injured & 1.591 & $0.992-2.552$ & 0.054 & 0.625 & $0.2188-1.784$ & 0.380 \\
$\quad$ & & & & & \\
Head /Limb & 4.361 & $2.6183-7.265$ & $<.001$ & 4.277 & $1.9027-9.615$ & $<.001$ \\
Thorax-adbomen/Limb & 5.703 & $1.4529-22.387$ & 0.013 & 8.041 & $1.3568-47.648$ & $\mathbf{0 . 0 2 2}$ \\
$\quad$ Multiple site/ Limb & 2.852 & $1.4689-5.536$ & 0.002 & 4.075 & $1.4483-11.467$ & $\mathbf{0 . 0 0 8}$ \\
Circumstances : EDL/RTA & 0.742 & $0.437-1.262$ & 0.271 & 1.487 & $0.6367-3.475$ & 0.359 \\
Family size & 1.015 & $0.7851-1.312$ & 0.911 & - & & - \\
Accident time interval & & & & & & \\
$\quad$ 5 pm-8pm / 8am-4pm & 1.473 & $0.827-2.624$ & 0.188 & 1.719 & $0.8514-3.473$ & 0.131 \\
9 pm-00am / 8am-4pm & 1.106 & $0.528-2.315$ & 0.789 & 0.933 & $0.3466-2.514$ & 0.891 \\
1 am-7am /8am-4pm & 3.110 & $0.665-14.553$ & 0.150 & 1.446 & $0.1028-20.352$ & 0.785 \\
\hline
\end{tabular}

\section{Discussion:}

This study examined the general characteristic for children over a 4-year period. Several factors could explain the variability of injury rates reported in studies from different countries. The most significant factors are likely differences in data collection methods and in the population samples in terms of sociodemographic (age, sex, education, income), cultural (health care system), and lifestyle (health care-seeking behaviour) characteristics [2]. Compared with adults, children are susceptible to unintentional injuries, and the sorts and causes of unintentional injuries are closely related to children's ages and growth levels [6].

In our study, the most common mechanism of unintentional injuries was falls (70.5\%). Previous studies in the literature have shown frequencies ranging from $1 \%$ to $61 \%[7,8]$. These results may be related to children's poor balance and the onset of independent mobility [9].

Almost one-half of our children's injuries occurred within the home environment (45.4\%). In similar studies, the rate of occurrence at home ranged from $44 \%$ to $57 \%$, because children spend most of their time at home [1014]. Regarding the gender distribution, we found a higher incidence of unintentional injuries among boys, which corroborates several studies of unintentional injuries. This can be justified, among other factors, by their dynamic lifestyle and less controlled behaviour $[5,15,16]$. Some researchers showed that the occurrence of children's unintentional injuries has common pathogeny with children abuse and ignorance [17]. Indeed, the stage of development and children age, the likely nature of injury type and setting are associated with a child's stage of development. Early childhood is characterised by a rapid increase in physical growth and cognitive functioning during which children become more mobile, independent and keen to explore their immediate environment. For pre-school children, home accidents, such as burns, scalds and poisonings, are more prevalent since the home is where they spend most of their time.

As children become older and greater independence allows them to leave further from home, the risk of outdoor injury increases.

The road environment presents a particular hazard, with children at risk as pedestrians, cyclists and subsequently as young drivers [18]. Our paper noticed a small proportion of injuries related to road traffic accident (RTA). This can be explained by the fact that some accidents due to cycles are considered as related to everyday life injuries, even if it occurs in the road, as well as those happening on the road to school. This paper highlighted the vulnerability of child under 16, and stressed on the need of more attention. When it comes to risk factors, some factors are known to be associated to severe for of injuries, such as the age greater than 5 years old, the falls from heights and sport-related injuries. $[19,20]$. The presence of an adult especially the mother seems to be protective [20]. In this study, head trauma, thoraco-abdominal and multiple sites are significantly associated to a moderate/severe form of injury.

Social protection is a right of the child, affirmed by Article 26 of the Convention International Rights of the Child, ratified by Morocco on June 21, 1993. This article has: 
*1. "States Parties recognize the right of every child to benefit from social security, including social insurance, and take the necessary measures to ensure the full realization of this right in accordance with their national legislation.

*2. Benefits should, where appropriate, be granted on the basis of means and the situation of the child and of those responsible for the maintenance of the child, as well than any other consideration relating to a request for benefits presented by or on behalf of the child. "[21].

The parent's educational conditions are one of the factors of health inequalities among children [22]. Maternal education is generally recognized as a strong determinant of child survival. When it is taken into account, the impact of father's education on child survival is considered to be less important, or is used as an indicator of the socioeconomic level of the household [23]. On the other hand, family homes are be thought of as a safe place for children, while the majority of the unintentional injuries happen at home or its surrounding. Thus, the need to prevent injuries might not be considered enough [24]. Furthermore, the Moroccan health insurance regime is a limited, unequal and fragile system with a total of just over 8.5 million people covered, which makes patient's care bellow expectations [21].

\section{Conclusion}

This study provides a general overview of unintentional accidents among children in Morocco, and allows a better understanding of the common mechanisms and patterns of injuries, essential to predict, treat and prevent unintentional injuries. Some factors are associated to a severe form of injuries and should be taken in account to establish a preventive policy. The mother's education, the social environment, the medical insurance should be improved as well as the homes and transportation means. The caregivers and health care providers are also a part of this prevention.

\section{References}

1. L.Roy, F.Léveillé, Les traumatismes non intentionnels en Chaudière-Appalaches Direction de santé publique et de l'évaluation QUEBEC (2009)

2. G. Santagati, L. Vezzosi, and I. F. Angelillo, MPH. Unintentional Injuries in Children Up to Six Years of Age and Related Parental Knowledge, Attitudes, and Behaviors in Italy

3. M. Craig Watson G. Errington Preventing unintentional injuries in children: successful approaches (2016)

4. X. Jianga, Y. Zhangb, Y. Wangb, B.Wanga, Y. Xub, Lei Shangb, An analysis of 6215 hospitalized unintentional injuries among children aged 0-14 in northwest China (2009)
5. The jamovi project (2021). jamovi. (Version 1.6) [Computer Software]. Retrieved from https://www.jamovi.org

6. Li, Yang, Wei, Min., Actuality of children unintentional injury study. Chin. J. Nurs. 41 (12), 1136-1138. (1998)

7. B. Chevallier, M. Sznajder, M-H. Bonnin, Prévention des accidents de la vie courante des enfants: approche épidémiologique et /ou communautaire? Archives de pédiatrie;18:1-2. (2011)

8. LR. Inbaraj, A. Rose, k .George A. Bose, Incidence and impact of unintentional childhood injuries: a community based study in rural South India. India J Pediatr; doi: 10.1007/s12098-016-2260-6 (2016)

9. M. Peden, K. Oyegbite, O. Smith, World report on child injury prevention. Geneva: World Health Organization,

10. A. Barat, M. Craig Watson, C.A. Mulvaney, accidents in children at Dakar: A study of 555 cases. Journal de pédiatrie et de puériculture 28 , 217-222. (2015)

11. M. Rafai, N. Mekaoui, N. Chouaib, L. Belyamani, A. El Koraichi, S. Ech-Cherif El Kettani et al. Epidemiology of severe domestic accidents of children admitted in pediatric intensive care unit of Children Hospital of Rabat-Morocco. Pan Afr Med J.; 20:28. (2015)

12. M.HU, G.HU Qing, ZQ. SUN, and X. HE, Epidemiological Survey of the Prevalence of Non-fatal Injury among Children Aged 5-14 Years in ChinaBiomed Environ Sci, 25(4): 407412. ( 2012)

13. EF Halawa, A Barakat, HI Rizk, EM Moawad. Epidemiology of non-fatal injuries among Egyptian children: a community-based crosssectional survey. BMC Public HealthDec 17;15: 1248. (2015)

14. G. Santagati, L. Vezzosi, IF. Angelillo. Unintentional Injuries in Children Up to Six Years of Age and Related Parental Knowledge, Attitudes, and Behaviors in Italy. J Pediatr; 177:267-72. (2016)

15. H. Oubejja, R. Razine, H. Zerhouni, M. Erraji, Ettayebi F, Soulaymani A. Epidemiological profile of child victims of home related injuries, hospitalized inchildren's hospital of Rabat, Morocco. International Journal of Innovation and Scientific Research. ISSN 2351-8014 Vol. 17 No. 1 Aug., pp. 69-76. (2015)

16. LA. Del Ciampo, IRL. Del Ciampo, Uninentional Injuries Among Children: An Observational Study in a Basic Health Unit in Ribeirão Preto, Brazil. American Journal of Pediatrics. Vol. 2, No. 5, 2016, pp. 19-22. doi: 10.11648/j.ajp.20160205.11 (2016)

17. X. Jianga, Y. Zhang b, Y. Wang b, B. Wanga, Y. Xub, L. Shang, An analysis of 6215 hospitalized unintentional injuries among children aged 0-14 in northwest China(2010) 
18. M. Craig, W. Gail Errington Preventing unintentional home injuries among children: exploring the perceptions of Iranian health professionals. Primary Health Care Research \& Development (October 2019)

19. C. Macarthur, X. Hu, DE.Wesson, \& PC. Parkin. Risk factors for severe injuries associated with falls from playground equipment. Accident Analysis \& Prevention, 32(3), 377-382 (2000)

20. EJ. Bardón Cancho, C.Arribas Sánchez, A. Rivas García, J. Lorente Romero, P.Vázquez López, \& R.Marañón Pardillo. Management and serious risk factors associated with unintentional injuries in paediatric emergencies in Spain. Anales de Pediatría (2020)

21. La protection sociale au Maroc Revue, bilan et renforcement des systèmes de sécurité et d'assistance sociales

22. T.Ravelomanana' M. Rakotomahefa, N. Randrianaivo, SH. Raobijaona, H. Barennes, Mother's educational level and children's illness severity in the emergency unit of JosephRaseta-Befelatanana Hospital. What kind of implications? (2020)

23. B. Bayda, instruction des parent et survue des enfants au BURKINA FASO : Groupement d'intérêt scientifique EHESS-INED-INSEEORSTOM-PARIS VI (1998)

24. M. Hu, GQ. Hu, ZQ Sun, X He. Epidemiological survey of the prevalence of non-fatal injury among children aged 5-14 years in China. Biomed Environ Sci 2012;25:40712.(2012) 